\title{
Silicone Gel Compounds for Non-Linear Stress Grading Applications
}

\author{
Sebastian Wels ${ }^{1}$, Johann Bauer ${ }^{2}$, Jens Lambrecht ${ }^{3} \&$ Albert Claudi $^{1}$ \\ ${ }^{1}$ University of Kassel (Kassel, Germany) \\ ${ }^{2}$ Merck KGaA (Darmstadt, Germany) \\ ${ }^{3}$ Wacker Chemie AG (München, Germany) \\ wels@uni-kassel.de
}

\begin{abstract}
The paper deals with a field-grading insulation using silicone gel as main material/binder. Additives, which provide the nonlinearity of the compounds, consist of a support material with metal oxide coating. The nonlinear electrical properties were adjusted by the addition of different dopants. In this investigation the fundamental properties of new non-linearconductive materials (NLCM) were examined. One aim of the investigation is to attribute the macroscopic electrical behavior to the respective doping. For a typical compound, field calculations for a cable joint model are presented and compared to a conventional design without NLCM.
\end{abstract}

\section{Introduction}

It is state of the art of the design of all insulation systems, to definitely avoid local field strength peaks. Such systems represent a rather mechanically complex, costly solution. A new design solution approach is an innovative insulating material, which shows a reduction of the specific electrical volume resistivity with increasing external field strength whereas they remain totally insulating at lower field strength (non-linearconductive materials - NLCM). An externally imposed increased field strength causes a local increase of the conductivity of the NLCM. Thus, the resulting maximum field strength in the material is reduced. The expected commercial and technical advantages are [1]:

- Lower complexity of the insulation system in comparison with existing solutions,

- Elimination of processing steps in manufacturing and installation, which finally leads to:

- Reduced total costs of the insulation.

An optimum material cannot be defined. Rather, the NLCM used, should be adapted to the respective application. For this purpose, five differently doped additives were examined. Afterwards the measured electrical properties were used to execute a field strength simulation of a simple cable joint model for medium voltage applications.

In the following, exemplary results of all materials are presented to clarify significant differences only.

\section{Material}

The field-grading insulation material uses a 2-component silicone gel as main material. The used additives are inorganic substrates with metal oxide coating (Figure 1) and different dopants hereafter called A, B, D, F and G. The nonlinear electrical properties are adjusted by the introduction of dopants. The evaluation of the different additives is executed with a filling degree of 10 vol. $\%$.

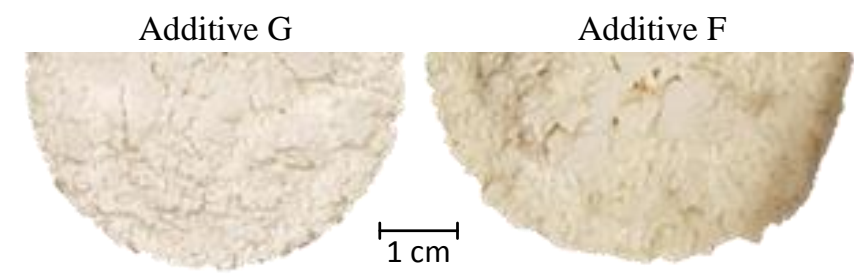

Figure 1. Macroscopic view of additive G and F.

Due to the absorption of moisture the additives tend to form a lumpy structure. It is therefore essential to dry the powders before processing.

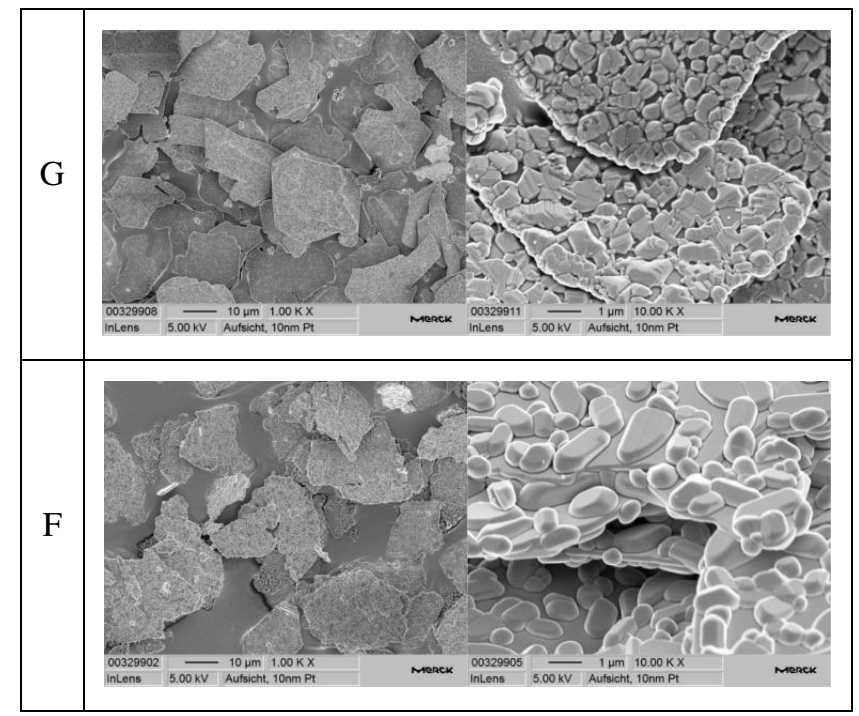

Figure 2. SEM image of filler particles.

Figure 2 shows the particles with a magnification of $1 \mathrm{k}$ (left) and $10 \mathrm{k}$ (right). The scanning electron microscope (SEM) images show the particles of additives F and G. According to the SEM image of all fillers the selected doping seems to have a 
significant influence on the formation of the boundary layer of the particles.

At a magnification of $1 \mathrm{k}$, the platelet-shaped structure is clearly visible. The particles have irregular sizes in the range between $5 \mu \mathrm{m}$ and $35 \mu \mathrm{m}$.

The image with $10 \mathrm{k}$ magnification illustrates the granular coating of the particles. Material $\mathrm{G}$ shows a flatter and denser surface compared to material $\mathrm{F}$.

\section{Fundamental Measurements}

To simulate the electric field distribution within a larger insulation arrangement, the specific conductivity and the relative permittivity of the insulating material are required. Since the used material changes its specific conductivity a field strength dependent consideration of this value is necessary. Contrary to the temperature, especially the humidity has a significant influence on the electrical properties of the considered NLCM. Consequently, this influence should also be considered.

\subsection{Conditioning}

The conditioning of the samples was carried out at an ambient temperature of $(25 \pm 3){ }^{\circ} \mathrm{C}$ over a period of 500 hours. The relative humidity in the desiccator was adjusted with saturated salt solutions or silica gel:

- Magnesium nitrate ( $\approx 50 \%$ r. h.),

- Potassium sulphate (>90\% r. h.),

- Silica gel (<20\% r. h.).

\subsection{Specimens}

The investigation of the compounds was performed under a nearly homogeneous electric field. For this purpose, a plate-plate electrode arrangement was used, which was casted with the different compounds. The edges are shaped according to a Rogovski-profile with a gap of $\mathrm{s}=2 \mathrm{~mm}$. The effective diameter was $d=18 \mathrm{~mm}$ (Figure 3 ). The field distortion at the electrode edges was neglected. Four specimens were manufactured and measured for each material.

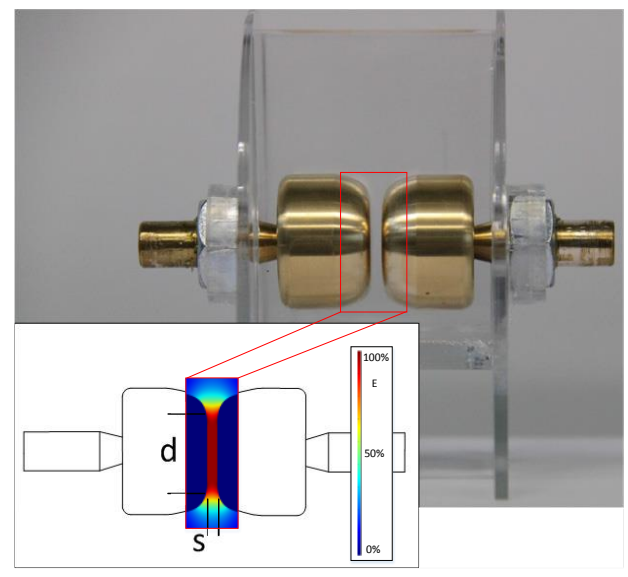

Figure 3. Electrode arrangement [2].

\subsection{Test Setup}

The following section describes the procedures, which were used to determine the specific conductivity and relative permittivity. All measurements were carried out at $25{ }^{\circ} \mathrm{C}$ and under dry $(<20 \%$ r.h. $)$, normal $(\sim 50 \%$ r. h. $)$ and humid (>90\% r. h.) climate.

The most important feature of the NLCM is the dependency between the imposed field strength $\mathrm{E}$ and the resulting current density $\mathrm{J}$, the $\mathrm{J}$-E-characteristic, which was measured under stepped DC in a circuit according to Figure 4. The current was measured five seconds after adjusting the voltage. In order to avoid deterioration of the specimens, a maximum current density was limited to $0,004 \mathrm{~A} / \mathrm{m}^{2}$.

In general, repeated measurement of a sample lead to the same J-E-characteristic. An exception to this is the first measurement, where polarization and orientation processes lead to a slightly different J-E-characteristic. In the following, therefore, always the second measurement of the compound is shown.

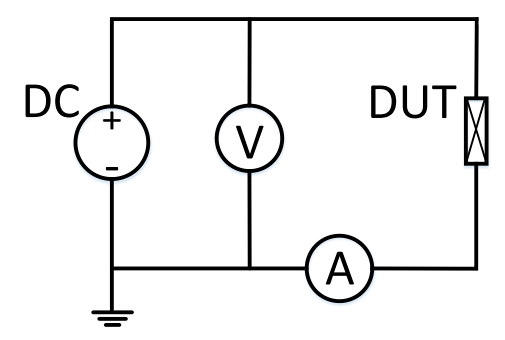

Figure 4. Measure setup J/E-characteristic [1].

For a normalized presentation of the results the current density (J) and the DC-field-strength (E) are calculated from the geometry of the test specimen according to formula (1) and (2),

$$
\begin{aligned}
& E=\frac{V}{s} \\
& J=\frac{I}{\pi \cdot r^{2}}
\end{aligned}
$$

where $\mathrm{V}$ is the voltage, $\mathrm{I}$ is the current, s the electrode distance and $r$ the effective electrode radius.

To determine the relative permittivity, the capacity of the test specimens in the filled and unfilled state was measured. Afterwards the relative permittivity could be calculated with formula (3). With the used measurement device Agilent E4980A LCR Meter, the capacitance of the test specimens was measured at a frequency of $1 \mathrm{kHz}$ and a voltage of $2 \mathrm{~V}$,

$$
\varepsilon_{r}=\frac{C_{f}}{C_{e}}
$$

where $\mathrm{C}_{\mathrm{f}}$ is the capacity of the filled and $\mathrm{C}_{\mathrm{e}}$ the capacity of the empty device. 


\subsection{Results}

\section{1) J/E-characteristic at normal conditions}

$\mathrm{J} / \mathrm{E}$-characteristics of different compounds are presented in Figure 5 for normal conditions $\left(25^{\circ} \mathrm{C}, 50 \% \mathrm{r}\right.$. h. $)$. A reference point is defined as the intersection of the tangent at the operating point $\left(0.001 \mathrm{~A} \mathrm{~m}^{-2}\right)$.

As already mentioned, an optimal material behavior cannot be determined without the definition of a specific application or stress level. Thus, an adjustability of the material properties with the introduction of different dopants may become important. Figure 5 shows the arithmetic mean values of the data obtained from all specimens of various compounds. The J-Echaracteristic show significant differences. Compound $\mathrm{F}$ reaches the current limit at $12.5 \mathrm{kV} / \mathrm{mm}$, while compound B is already at $1 \mathrm{kV} / \mathrm{mm}$ at the full-scale current value. Thus, it seems to be possible to control the respective material behavior by optimization of the doping.

It is noticeable that the characteristics with a higher reference point show a significantly lower nonlinearity. This behavior does not have to be negative, but an adjustability of the nonlinearity would expand the possible application range.

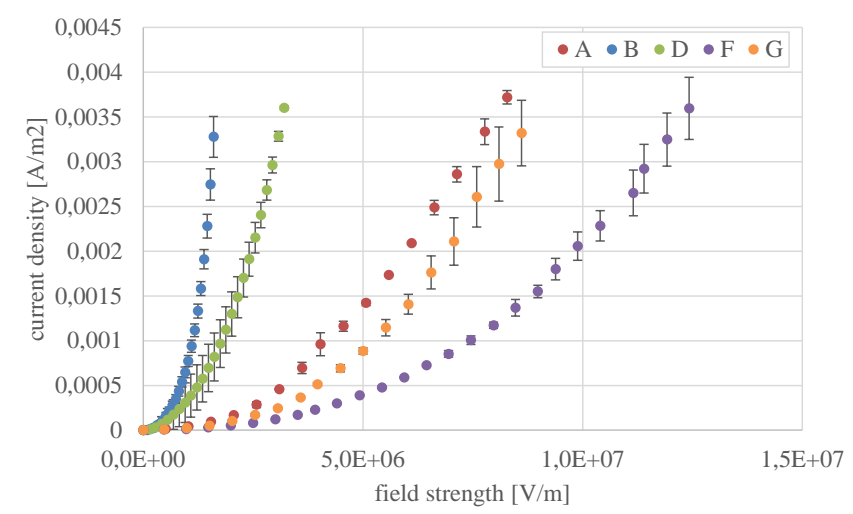

Figure 5. J/E- characteristic of all compounds $\left(25^{\circ} \mathrm{C}, 50 \%\right.$ r. h. $)$.

\section{2) Moisture influence on J/E-characteristic}

In addition to the material properties under normal conditions, their evaluation at changing ambient conditions is essential. The ambient humidity has a significant influence on the J/E-characteristics of the NLCM (Figure 6). Nevertheless, there are also clear differences between the materials examined. While the compound with additive A shows a slight shift of the $\mathrm{J} / \mathrm{E}$-characteristic only, the compound with additive $\mathrm{F}$ shows larger changes. An ambient humidity of $<20 \% \mathrm{r}$. h. leads to almost linear characteristics.

Since the only difference between the additives is the type and amount of doping, the different responses to moisture are also due to these differences in doping. Thus, some doping elements seem to favor water absorption.

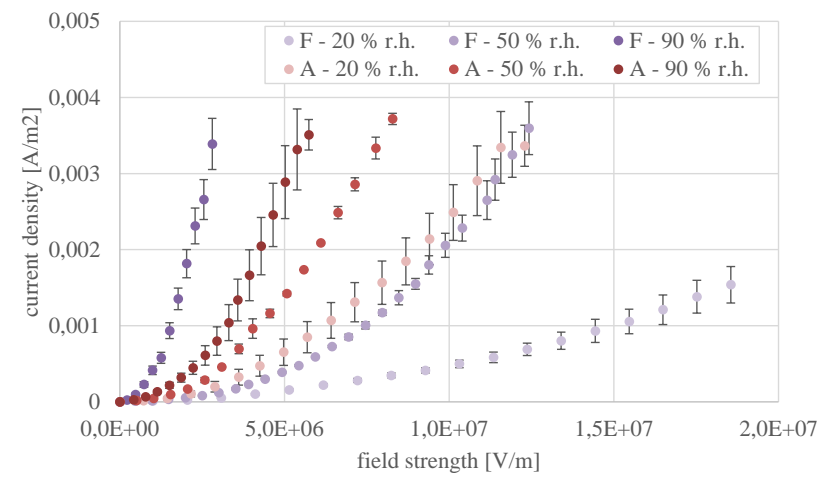

Figure 6. Moisture influence on J/E-characteristic of compounds with additive $\mathrm{A}$ and $\mathrm{F}$.

\section{3) Relative Permittivity}

Figure 7 shows the calculated relative permittivity. Obviously, the chosen dopants affect the permittivity. Especially the compound with filler B shows a greatly increased relative permittivity.

Furthermore, the relative permittivity increases with increasing water content in the insulating material. This increase could be explained by the high polarizability of the stored water molecules.
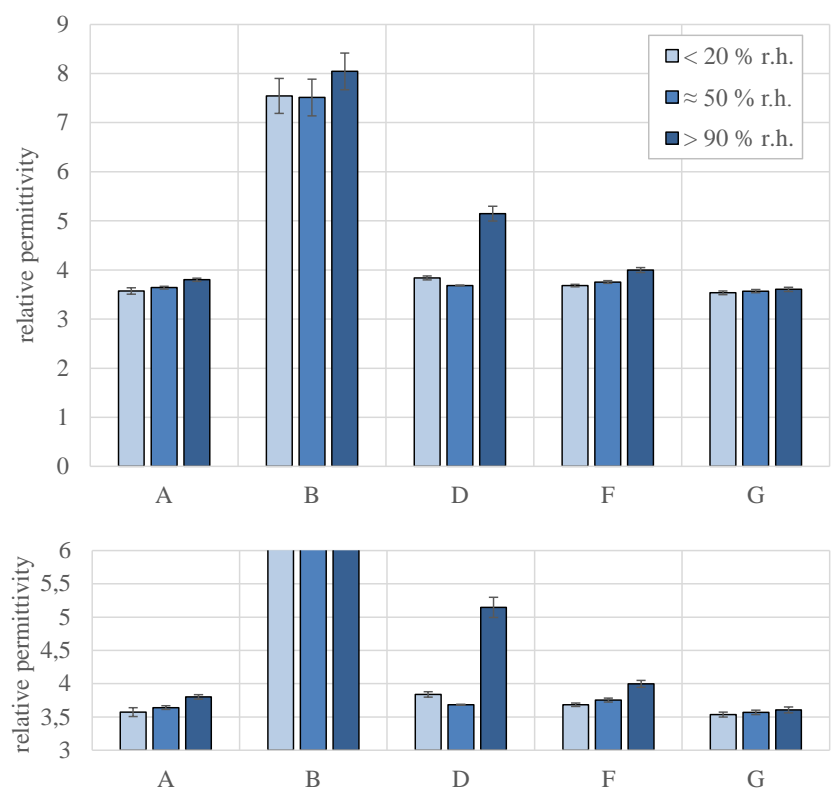

Figure 7. Relative permittivity (above) and detail view of A, D, F and $\mathrm{G}$ (below).

\section{Model Cable Joint}

Voluminous field grading insulations can be used in for a variety of applications. A typical field of application are cable joints in the medium and high voltage range. Especially this field of application has particularly high demands on the used insulation material. In the following the influence of the examined compounds as well as that of the moisture in the field distribution is evaluated by the simulation. For this purpose, a simple cable joint geometry was first designed and cumulatively considered using COMSOL Multiphysics®. 


\subsection{Modeling and Discretization}

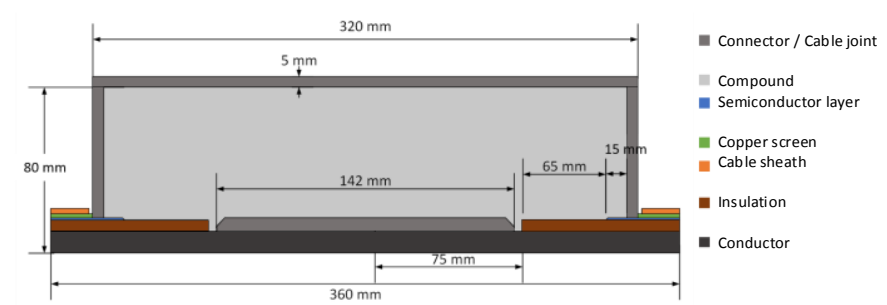

Figure 8. Model of a medium-voltage cable joint.

For the 3D-object of the cable joint, the rotationally symmetric arrangement can be used to reduce the simulation object to a 2D rotationally symmetric geometry.

Figure 8 shows the geometry, which was used to execute the calculation. On the right side of Figure 8 the individual materials of the cable and joint are listed. The voltage of $24 \mathrm{kV}\left(2 \mathrm{U}_{0}\right)$ is applied to the inner conductor. The aluminum housing was set to earth potential. The discretization of the model is shown in Figure 9.

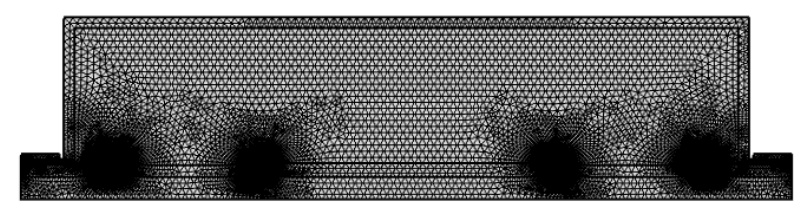

Figure 9. Discretization.

To describe the insulation material, COMSOL Multiphysics ${ }^{\circledR}$ requires the field-strength-dependent conductivity and the relative permittivity. The field-strengthdependent conductivity characteristic is stored in the software by piecewise cubic interpolation between the measured characteristic points and a linear extrapolation.

\subsection{Results of the Electrical Field Simulations}

\section{1) Influence of additives}

Figure 10 shows the electric field strength distribution in the joint at an applied voltage of $24 \mathrm{kV}\left(2 \mathrm{U}_{0}\right)$ under normal conditions $\left(25{ }^{\circ} \mathrm{C}, 50 \%\right.$ r. h.). Pure silicone gel (left) and compounds with additives A (middle) and B (right) were calculated as insulating materials. The field strength scale has been set to the respective maximum field strength. This occurs, as Figure 10 (bottom) shows, at the settling edge of the semiconductor layer of the cable.

The expected difference between the insulation materials is the reduced maximum field strength (right above field strength scale). According to this approach, the compound with additive $B$ significantly reduces the field strength and thus provides the best result. However, the selected scaling range doesn't show in which area the field grading takes place.
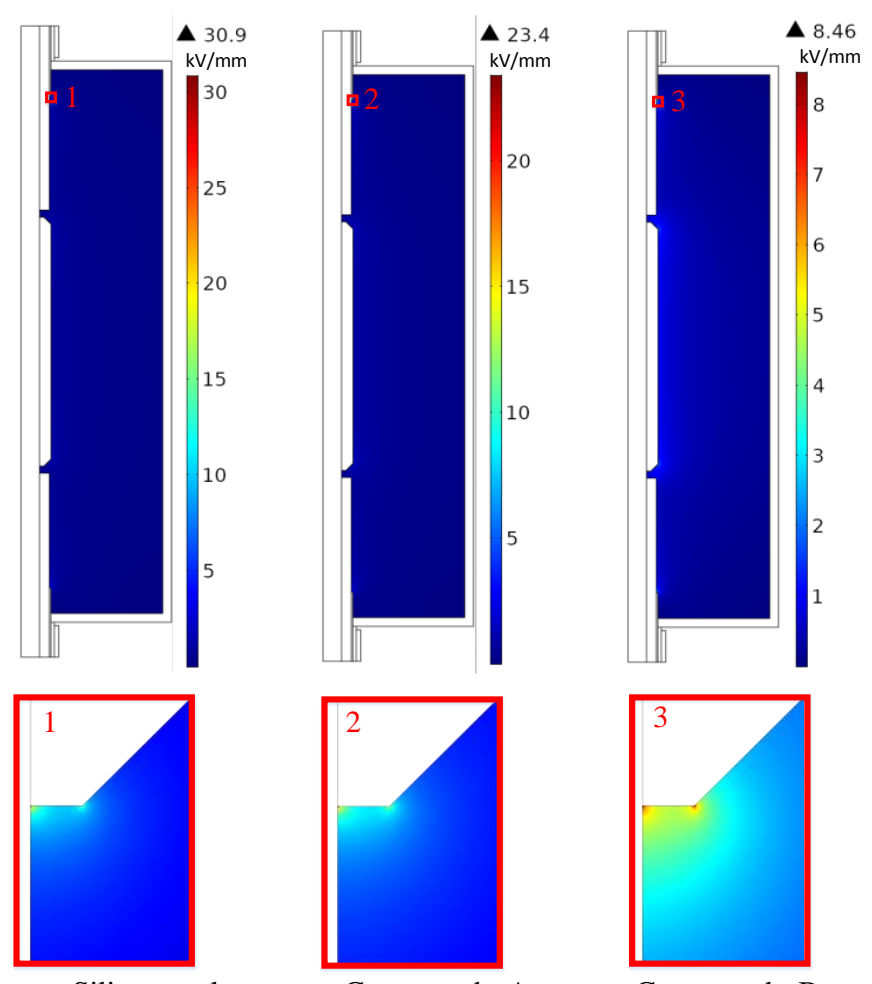

Silicone gel

Compound - A

Figure 10. Electric field strength distribution in the cable joint under the use of silicone gel and compound A and B (scale normalized to respective maximum field strength).

In the following illustration (Figure 11), the field strength scales are adjusted to the reference point of the two different compounds. For the compound with additive A, a reference point of $3 \mathrm{kV} / \mathrm{mm}$ was specified, for the compound with additive $\mathrm{B}$, the scale was set at $1 \mathrm{kV} / \mathrm{mm}$. This representation allows to show the working scale of the compound; in all dark red areas (field strength greater than the reference-point-fieldstrength) the specific volume resistance is reduced.

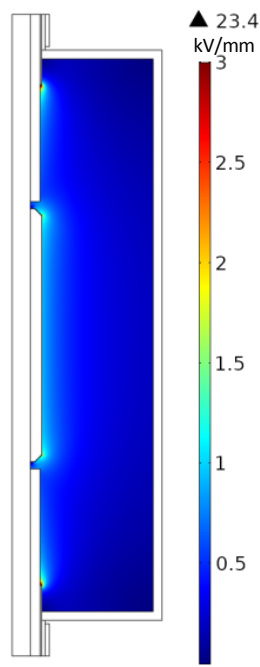

Compound - A

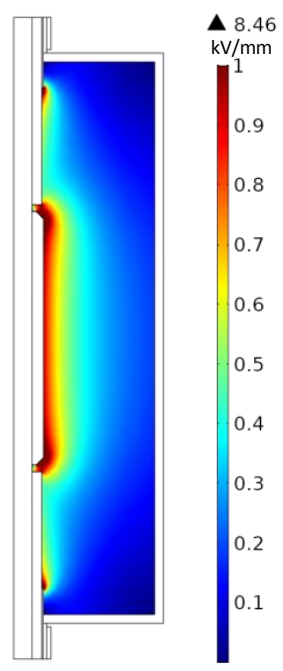

Compound - B

Figure 11. Electric field strength distribution in the cable joint under the use of compound A and B (scale normalized to respective switching point) 
It becomes clear, that the compound with additive $\mathrm{B}$ requires a much larger volume to reduce the field strength. This behavior can be understood to be less suitable, especially for high-voltage applications, since the significantly higher field strengths can lead to a higher conductivity of the complete insulation volume. Thus, for applications with higher field strengths, additive A would be more suitable.

\section{2) Influence of moisture}

The influence of moisture on the cable joint arrangement was simulated with additive $\mathrm{A}$ and $\mathrm{F}$ at $50 \% \mathrm{r}$. h. and $90 \% \mathrm{r}$. h. (Figure 12). The test voltage again was $24 \mathrm{kV}\left(2 \mathrm{U}_{0}\right)$. The field strength scales are fixed to the switching point of the respective compound at the respective humidity.

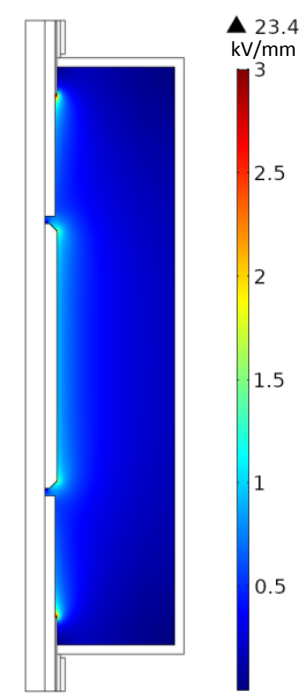

Compound - A - $50 \%$ r. h.

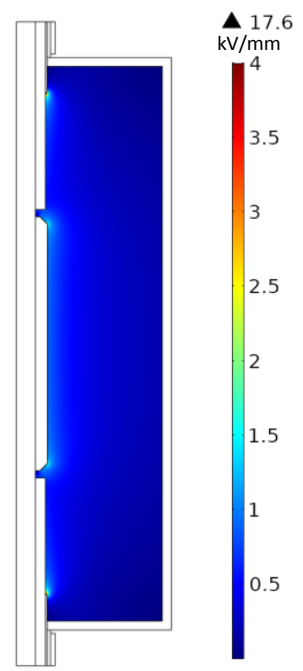

Compound - F - $50 \%$ r. h.

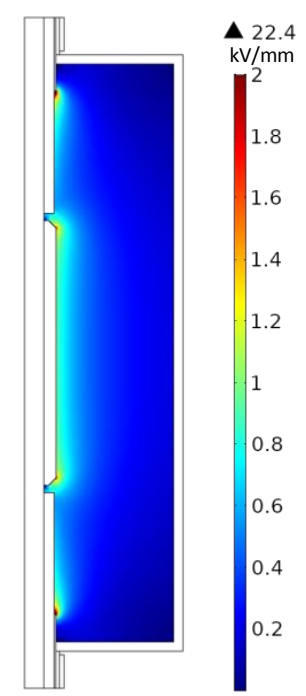

Compound - A - $90 \%$ r. h.

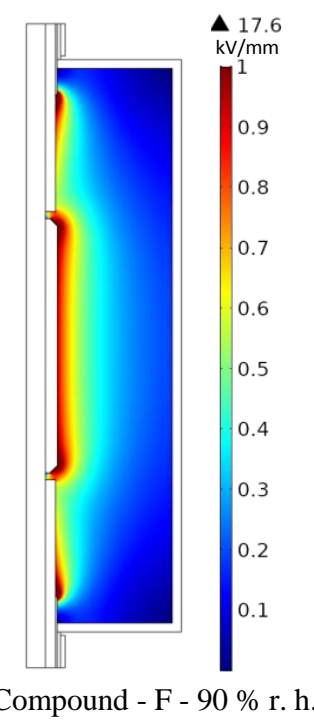

In the behavior of compound $\mathrm{F}$, clear differences are showing up. A large shift of the reference point from $4 \mathrm{kV} / \mathrm{mm}$ to $1 \mathrm{kV} / \mathrm{mm}$ takes place, consequently a large change of the working area occurs.

Small changes, such as those of compound A, are reasonable and can be considered when insulation systems with fieldcontrolling insulating materials are developed. The large changes of compound $\mathrm{F}$ are not sustainable and need to be significantly improved if humidity cannot be avoided in the application.

\section{Conclusion}

In this publication non-linear field strength grading insulating materials were considered. Silicone gel was used as main material/binder. The additives, which cause the nonlinearity, consist of a support material with metal oxide coating. The nonlinear electrical properties were adjusted by the introduction of different dopants.

In general, an optimum material behavior cannot be defined yet. Rather, the NLCM used, should be adapted to the respective application. For this purpose, the relationship between electrical properties and various modified additives was investigated. Within this examination it was shown that different NLCM could be adapted to the respective application by modification. For further investigations, it should be noted that the adjusted additives also react differently to different ambient conditions. This has been demonstrated by the influence of the relative ambient humidity.

In a second step, the measured electrical properties were used to execute a field strength simulation of a simple cable joint model for medium voltage applications. The significant influence of the different J-E-characteristics on the field strength distribution could be clearly shown. Depending on the imposed field strength, the NLCM compound must be adapted to the respective application in order to define an optimum operating range.

An important point, which should be considered in followup investigations, is the modification of the non-linearity of the $\mathrm{J}$-E-characteristic in high field strength ranges.

\section{References}

[1] S. Wels, J. Lambrecht and A. Claudi, "Silicone Gel Compounds for Medium and High Voltage Insulation with Nonlinear Stress Grading - State of Art", 6th Conference on Silicone Insulation, Burghausen, pp. 103- 107, 2017.

[2] A. Austermühl A. Claudi, "Investigation on the electrical behavior of metal oxide pigments filled silicone gels", 18th International Symposium on High Voltage Engineering (ISH), Seoul, Korea, 2013.

Figure 12. Electric field strength distribution in the cable joint under the use of compound $\mathrm{A}$ and $\mathrm{F}$ with different moistures (scale normalized to respective switching point).

The reference point of compound A shifts from $3 \mathrm{kV} / \mathrm{mm}$ to $2 \mathrm{kV} / \mathrm{mm}$. The increased humidity has only a small influence on the resulting field strength distribution. 